\title{
Development of a Ferrite-Based Electromagnetic Wave Detector
}

\author{
Muhammad Hanis Bin Zakariah ${ }^{1, *}$, Poppy Puspitasari ${ }^{2}$, Nur Azliza Ahmad \\ ${ }^{1}$ Department of Electrical and Electronic Engineering, Universiti Teknologi PETRONAS, Bandar Seri Iskandar, 31750 \\ Tronoh, Perak Darul Ridzuan, Malaysia \\ ${ }^{2}$ Department of Mechanical Engineering, Engineering Faculty, Universitas Negeri Malang, Semarang St. No 5, Malang, \\ East Java, Indonesia, 65140 \\ ${ }^{3}$ Faculty Engineering, Mahsa University, Saujana Putra Campus, 46210 Jenjarom, Selangor \\ *mhanisz@gmail.com
}

\begin{abstract}
Direct detection of hydrocarbon by an active source using electromagnetic (EM) wave termed Sea Bed Logging (SBL) has shown very promising results. However, currently available electromagnetic wave technology has a number of challenges including sensitivity and lapsed time. Our initial response to this issue is to develop a ferrite-based EM wave detector for Sea Bed Logging (SBL). Ferrite bar and copper rings in various diameters were used as detector 1 (D1). For Detector 2 (D2), toroid added with copper wires in different lengths at the centre of it were used. The first experiment is to determine the inductance and resistance for both detectors by using LCR meter. We obtained the highest inductance value of 0.02530 $\mathrm{mH}$ at the ferrite bar when it was paired with a $15 \mathrm{~cm}$ diameter copper ring and $0.00526 \mathrm{mH}$ for D2 using a $100 \mathrm{~cm}$ copper wire placed at the centre of the toroid. The highest resistivity for D1 was measured at ferrite bar paired with a $15 \mathrm{~cm}$ diameter copper ring and $1.099 \Omega$ when using $20 \mathrm{~cm}$ length of copper wire. The second interest deals with voltage peak-to-peak (Vp-p) value for both detectors by using oscilloscope. The highest voltage value at the ferrite bar of D1 was $25.30 \mathrm{mV}$. While at D2, the highest voltage measured was $27.70 \mathrm{mV}$ when using a $100 \mathrm{~cm}$ copper wire. The third premise is the comparison of sensitivity and lapsed time for both detectors. It was found that D1 was $61 \%$ more sensitive than D2 but had higher lapsed time than D2..
\end{abstract}

Copyright (C) 2017Journal of Mechanical Engineering Science and Technology

All rights reserved

Keywords: Sea Bed Logging, Detector, Sensitivity,Lapsed time

\section{Introduction}

In SBL, a mobile horizontal electric dipole (HED) source and an array of seafloor electric field receivers are utilised. The transmitting dipole emits a low frequency electromagnetic signal that propagates into the seabed [1]. The array of sea floor receivers measures both the amplitude and the phase of the received signal directly from the transmitter, and waves reflected and guided from the seabed. The received signal depends on the resistivity structure beneath the seabed [2]. Characterisation and detection of the reservoir using SBL are based on the electrical conductivity consisting in all geological media. The principle of conductivity differencein geological media is applied in this technology [3]. Besides its advantage in segregating a resistivity in a non-conductive layer and conductive formation beneath the sea floor, the electromagnetic method has a robustness characteristic against bad operating conditions such as high temperature and high pressure [4].

Many types of electromagnetic detectors have been developed for use in this type of hydrocarbon detection method. However, currently available detectors have a number of challenges and one of them is the reading instability that can result in a misinterpretation. This could lead to the loss of significant investments.

This paper describes the initial work to develop an EM detector with high sensitivity and short recovery time for SBL. The first part of this work is to compare the sensitivity for both detectors. Sensitivity of detectors is an important aspect for SBL method. In the previous work, electromagnetic detector was used to geophysically detect layer boundaries [5]. This workhas been done because difficulties exist in magneto telluric and geomagnetic depth sounding method; these methods are geophysical methods sensitive to mantle melt. The selection of detectors plays a major role to gain good detection results. Since sensitivity was taken into account, another aspect that needs to be considered is lapsed time or recovery time. 
The second premise dealt in this work is the determination of recovery time. In detail, EM detector for SBL needs a higher sensitivity with a shorter lapsed time that can prevent any error in SBL's further data processing. EM detection system used to detect buried metallic in the soil [6] found errors in the data caused by uncertainties in sensor height. As a result, a fluctuation in data occurred. ] These two parameters are important for sensitive EM detectors because with the decreasing of recovery times, the efficiency of the detector will increase. Output of the EM wave detected was not affected by a pile-up caused by incoming EM wave. Now we can look thoroughly to the magnetic hysteresis element of the magnetic material used in this work. Hysteresis is a phenomenon in which the magnetic field lags behind the electric field [7]. Details about the hysteresis will be reviewed more comprehensively in the Results and Discussion section.

\section{Materials and methods}

A 20-turn toroidal transmitter with an aluminium rod placed in the centre of the toroid was used in this work. It was supplied by $5 \mathrm{MHz}$ frequency of square wave from a wave generator (Textronic AX493). The distance between the transmitter and the detector was fixed at one meter, as described in Figure 1 and Figure 2. The properties of the transmitter are summarised in Table 1. Two types of EM detectors were investigated. The responses from the detectors were then determined by measuring the induced voltage with an oscilloscope.

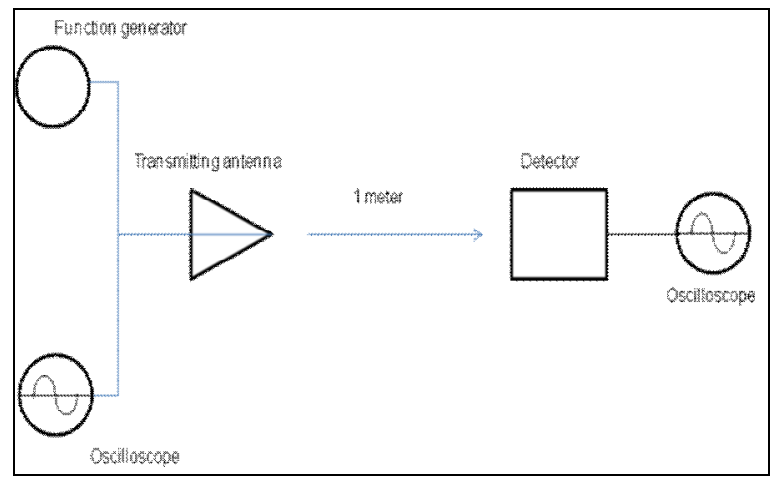

Fig. 1.Block diagram of transmitter-detector system

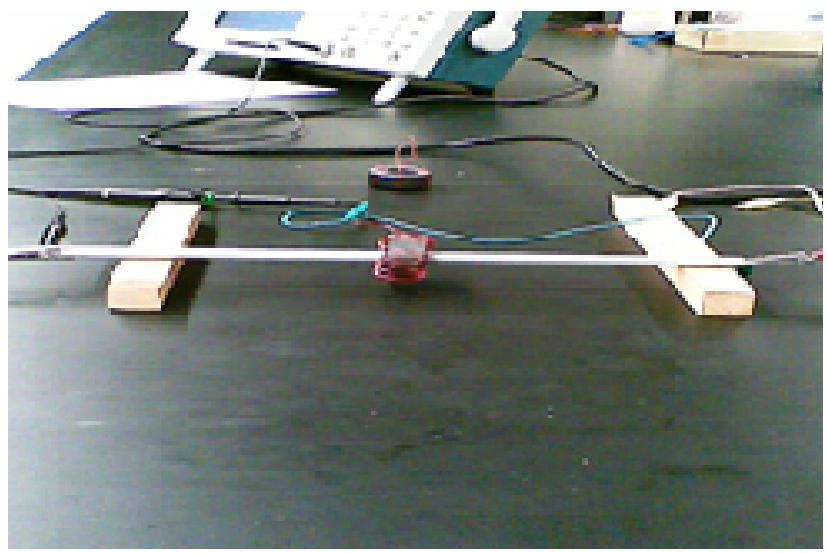

Fig. 2. Block diagram of transmitter-detector system

Table 1. Transmitter properties

\begin{tabular}{cc}
\hline Transmitter parameters & Properties \\
\hline aterial & Toroid with 20 turns and aluminium rod in the centre of the toroid \\
\hline Frequency & $5 \mathrm{MHz}$ \\
\hline Distance from detector & 1 meter \\
\hline Waveform & Square-shaped \\
\hline
\end{tabular}




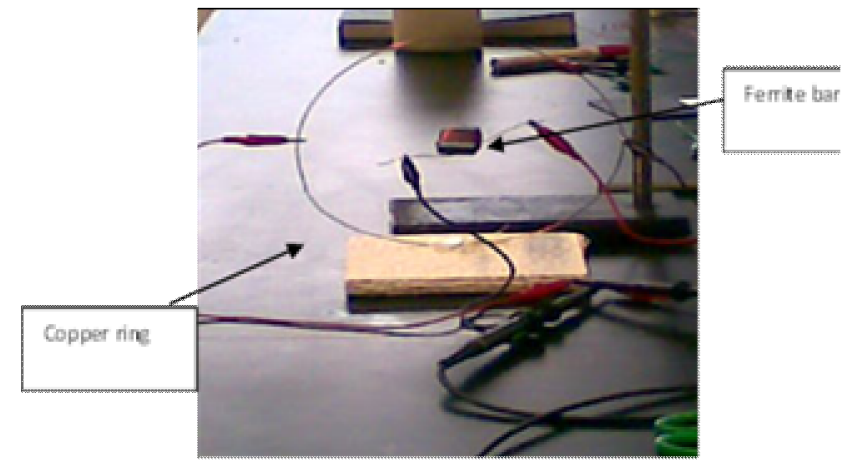

Fig. 3.Detector 1 (D1) set up consisting of a of a ferrite bar and copper wire

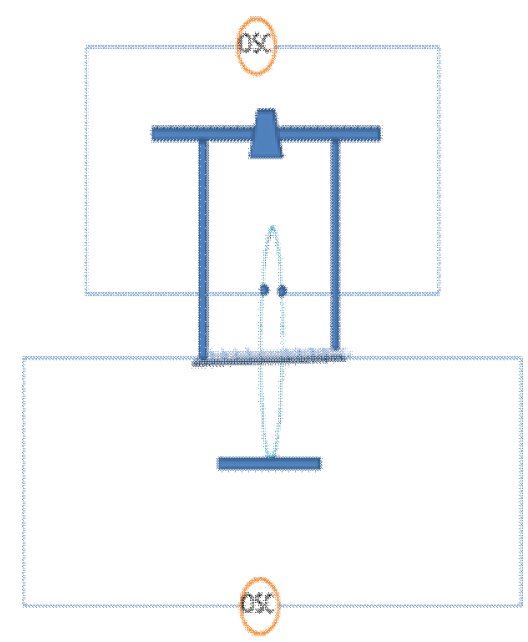

Fig. 4. Schematic diagram of D1

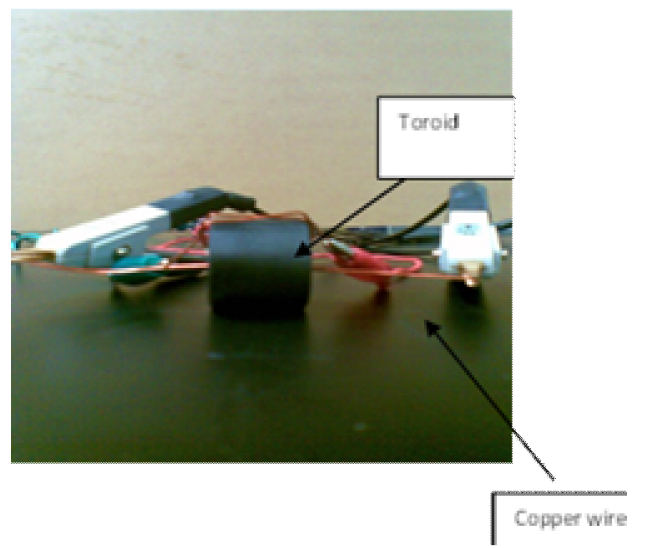

Fig. 5.Detector (D2) set up consisting toroid and copper wire

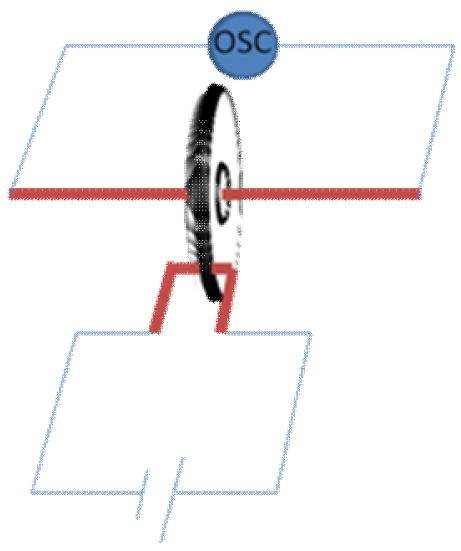

Fig. 6. Schematic diagram of D2

Table 2. Properties of ferrite material manufactured by ACME Ferrite Products Sdn Bhd (Source: www.acme.com.my)

\begin{tabular}{ccccc}
\hline Term & Symbol & Conditions & Value & Unit \\
\hline Initial Permeability & $\mu \mathrm{i}$ & $\begin{array}{c}10 \mathrm{kHz} \\
25^{\circ} \mathrm{C}\end{array}$ & $800 \pm 25 \%$ & \\
\hline $\begin{array}{c}\text { Max. Magnetic Flux } \\
\text { density }\end{array}$ & $\mathrm{Bm}$ & $\begin{array}{c}50 \mathrm{Oe} \\
25^{\circ} \mathrm{C}\end{array}$ & 3100 & Gauss \\
\hline $\begin{array}{c}\text { Residual Magnetic } \\
\text { Flux density }\end{array}$ & $\mathrm{Br}$ & $25^{\circ} \mathrm{C}$ & $1 / 00$ & Gauss $25^{\circ} \mathrm{C}$ \\
\hline Coercive Force & $\mathrm{Hc}$ & & 0.65 & \\
\hline Relative Loss factor & $\mathrm{tan} \mathrm{d} / \mu \mathrm{i}$ & $25^{\circ} \mathrm{C}$ & $0.15 \mathrm{MHz}$ & \\
\hline Electrical Resistivity & $\mathrm{p}$ & $\mathrm{DC}$ & $210^{-3}$ & $\Omega \mathrm{cm}$ \\
\hline $\begin{array}{c}\text { Temperature } \\
\text { Coefficient }\end{array}$ & & $25^{\circ} \mathrm{C}$ & $\leq 5$ & $10^{-6} \mathrm{~K}^{-1}$ \\
\hline Curie Temperature & $\mathrm{Tc}$ & & $>150$ & ${ }^{-1} \mathrm{C}$ \\
\hline Density & $\rho$ & & 5000 & $\mathrm{Kg}^{3}$ \\
\hline
\end{tabular}

Two types of EM wave detectors were selected in the present work. Figure 2 and Figure 3 show the arrangements of the two types of detectors used..In addition, their schematic drawings are presented in Figure 4 and Figure 5. 
Table 3. Properties of detector (D1 and D2).

\begin{tabular}{ccccc}
\hline & & Detector 1 (D1) & Detector 2 (D2) \\
\hline Copper wire & Shape & Ring-shaped with variable diameter & Wire \\
\cline { 2 - 4 } & Thickness & $0.8 \mathrm{~mm}$ with variable diameter & $0.8 \mathrm{~mm}$ with variable length \\
\cline { 2 - 4 } Toroid & Location & Placed vertically around the ferrite bar & \multicolumn{2}{c}{ Placed at the centre of toroid } \\
\hline & None & ID & 2.5mm \\
\cline { 3 - 4 } & & OD & Type D28 with constant electric \\
\cline { 3 - 4 } & & Type & source applied \\
\hline Ferrite Bar & Type D28 with 20 wind by copper wire (thickness & & None \\
& & & \\
\end{tabular}

Table 3. describes the properties of both detectors. The diameter of copper ring in D1 varies from $7.5 \mathrm{~mm}, 8.0 \mathrm{~mm}, 9.0 \mathrm{~mm}, 11.0 \mathrm{~mm}, 13.0 \mathrm{~mm}, 15.0 \mathrm{~mm}$, to $20.0 \mathrm{~mm}$.

Measurements of parameters for both detectors have been performed by using oscilloscope and LCR meter (Instex LCR-16). Parameters that we have put into consideration for both detectors were voltage peak to peak (Vp-p), inductance $(\mathrm{L})$, resistance, $(\mathrm{R})$ and recovery time (t). Moreover, measurements of $\mathrm{Vp}$-p have been carried out by using two oscilloscopes at the ferrite bar and copper ring; single function and both function operations were performed. Table 3 shows the clear method of operating oscilloscope during the measurement of Vp-p for D1.

Measurements of inductance (L) and resistivity (R) during detection period have been performed by using LCR meter at each ferrite bar and copper ring.

Detector 2 consisted of a small toroid as a magnetic feeder with a copper wire in the centre of it. A constant magnetisation was supplied by connecting $9 \mathrm{~V}$ dry-cell battery to the toroid. Copper wires $(0.8 \mathrm{~mm}$ thickness) with different set of length varying from $10 \mathrm{~cm}, 20 \mathrm{~cm}, 30 \mathrm{~cm}, 40 \mathrm{~cm}, 50 \mathrm{~cm}, 60 \mathrm{~cm}$, $70 \mathrm{~cm}, 80 \mathrm{~cm}, 90 \mathrm{~cm}$ to $100 \mathrm{~cm}$ were placed in turn at the centre of magnetic feeder. The measurement of V p-p for detector 2 have been taken by using oscilloscope at both ends of copper wire which had been placed at the centre of toroid during receiving an electromagnetic wave from the transmitter. By using the LCR meter, the inductance and resistance value have been determined during the detection period of EM wave.

Lapsed time measurement was performed to determine hysteresis effect by the following methods. When the detectors were exposed to EM wave, the signal detected at the oscilloscope showed a proportional response (due to the properties of the detector). Time taken by a signal detected at oscilloscope at the peak value (when the EM source transmitter was on) was dropped off to the base line when EM wave source (transmitter) is off were measured.

\section{Results and discussion.}

Measurements of sensitivity and lapsed time for the two detectors (D1 and D2) were carried out using a ferrite-core material [11]. Table 4 shows the results of Vp-p gained from D1 with the changing of diameter of the copper ring. The experiment conducted with oscilloscopes was divided into two sections, i.e. single function operation and both functions operation. During the single function operation of oscilloscope at the ferrite bar, the highest Vp-p value recorded was $45.60 \mathrm{mV}$, which was using a $15.0-\mathrm{cm}$-diameter copper ring. However, it was drastically decreased when both functions operation of oscilloscopes was operated.

Table 4. $V_{\mathrm{p}-\mathrm{p}}$ measurement method for D1using oscilloscope.

\begin{tabular}{ccc}
\hline & \multicolumn{2}{c}{ Oscilloscope function } \\
\cline { 2 - 3 } & Single operation & Both operation \\
\hline Ferrite Bar & Operated & Operated \\
\hline Copper ring & Non-operated & Operated \\
\hline Ferrite Bar & Non-operated & Operated \\
\hline Copper ring & Operated & Operated \\
\hline
\end{tabular}


Table 5. $\mathrm{V}_{\mathrm{p}-\mathrm{p}}$ values for D1

\begin{tabular}{ccccc}
\hline $\begin{array}{c}\text { Diameter of copper ring } \\
(\mathbf{c m})\end{array}$ & \multicolumn{2}{|c}{$\begin{array}{c}\text { Vp-p }(\mathbf{m V}) \text { single function } \\
\text { operation }\end{array}$} & \multicolumn{2}{c}{$\begin{array}{c}\text { Vp-p (mV) both function } \\
\text { operation }\end{array}$} \\
\hline & Ferrite & Ring & Ferrite & Ring \\
\hline 20.0 & 31.80 & 19.40 & 23.40 & 5.04 \\
\hline 15.0 & 45.60 & 17.10 & 7.79 & 7.79 \\
\hline 12.0 & 37.60 & 19.60 & 36.00 & 6.41 \\
\hline 11.0 & 24.40 & 19.60 & 25.30 & 14.50 \\
\hline 9.0 & 36.20 & 11.60 & 34.00 & 11.20 \\
\hline 8.0 & 37.80 & 9.43 & 10.70 & 10.70 \\
\hline 7.5 & 31.20 & 11.30 & 31.20 & 11.30 \\
\hline
\end{tabular}

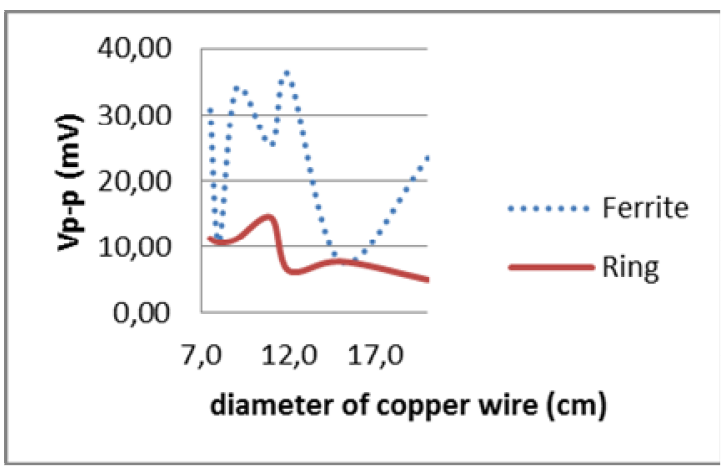

Fig. 7.Graph pattern for $\mathrm{Vp}$-p values for ferrite bar and copper ring from $\mathrm{D}_{1}$ during single operation of oscilloscope

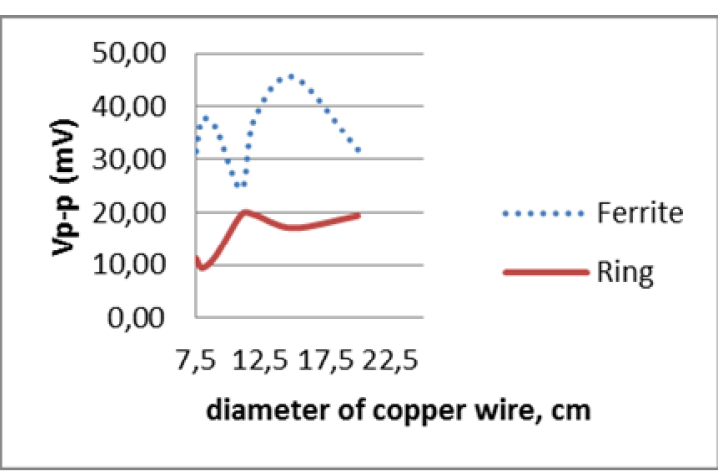

Fig. 8. Graph pattern for Vp-p values for ferrite bar and copper ring from D1 during both operations oscilloscope.

Table 6. L, R and Vp-p values for D1

\begin{tabular}{ccccccc}
\hline Diameter of copper ring $(\mathbf{c m})$ & \multicolumn{2}{c}{$\mathbf{L}(\mathbf{m H}) \mathbf{1} \mathbf{1 0}^{-2}$} & \multicolumn{2}{c}{$\mathbf{R}(\mathbf{\Omega})$} & \multicolumn{2}{c}{$\mathbf{V p - p}(\mathbf{m V})$} \\
\cline { 2 - 7 } & Ferrite & Ring & Ferrite & Ring & Ferrite & Ring \\
\hline 20.0 & 2.36 & 2.08 & 1.90 & 5.00 & 23.40 & 5.04 \\
\hline 15.0 & 2.53 & 1.86 & 8.97 & 7.24 & 7.79 & 7.79 \\
\hline 12.0 & 2.33 & 1.80 & 2.61 & 2.69 & 36.00 & 6.41 \\
\hline 11.0 & 2.35 & 1.66 & 3.02 & 3.20 & 25.30 & 14.50 \\
\hline 9.0 & 0.19 & 23.50 & 1.37 & 1.80 & 34.00 & 11.20 \\
\hline 8.0 & 2.35 & 1.63 & 1.91 & 2.55 & 10.70 & 10.70 \\
\hline 7.5 & 2.34 & 1.75 & 1.21 & 2.35 & 31.20 & 11.30 \\
\hline
\end{tabular}

In another work done on EM wave logging system for determining resistivity and dielectric constant of earth formations [8], it was proved that the resulting magnetic fields produced by electromagnetic induction from a high frequency alternating current in the earth formations surrounding the well bore were detected at the spaced receiver coil by sensing the induced currents or voltages in the receiver coil caused by the secondary currents flowing in the formations. Another former research found that when the copper ring and ferrite bar were exposed to an EM wave, their existing electric fields were found to be proportional with EM field [7]. The electric fields from ferrite bar and copper ring are supposed to enhance each other and lead to a higher value of Vp-p during both function operations than during single function operation of oscilloscope. However, when both function of oscilloscope test was conducted, there was a significant decrease in Vp-p at ferrite bar and copper ring to $7.76 \mathrm{mV}$ both. This result (Figure 3 and 4) showed that during detection period, the magnetic field from copper ring and ferrite bar could not enhance each other. Besides, the testing result of wider diameter of copper ring was expected to meet a threshold limit at 
a certain diameter of copper ring. The achieved threshold limit led to a conclusion that magnetic field at the ferrite bar is not affected by magnetic field of the copper ring.

Generally, the results of inductance $(\mathrm{L})$, resistivity $(\mathrm{R})$, and Vp-p values for $\mathrm{D} 1$ are presentedin Table 4 . The table shows the results obtained from measurements by using oscilloscope and LCR meter. Figure 7 and Figure 8 show the results of inductance and resistivity values obtained from D1.

As shown in Figure 7 and Figure 8, the average value of the inductance (L) and resistance (R) at the ferrite was higher than the one of $\mathrm{L}$ and $\mathrm{R}$ at copper rings.

In measurement of electromagnetic and electric field for D2, Figure 5 shows that resistivity was increased when the length of copper wire was increased as well. From the resistivity value, we concluded that an electric field existed. One important difference between electromagnetic poles and electric charges is that electric charges can be isolated, but magnetic poles always exist in pairs [9]. This was proven by inductance values indicatingthe strength of the magnetic field in D2.

Figure 12 shows the inductance values gained from LCR meter when electromagnetic field was applied. Inductance can be described as a measurement of how much magnetic energy stored in the detector [10]. The use of $100 \mathrm{~cm}$ copper wire in the centre of toroid generated the highest value of inductance $(0.00526 \mu \mathrm{H})$, resistivity $(0.9001 \Omega)$, and $\mathrm{Vp}-\mathrm{p}(27.70 \mathrm{mV})$

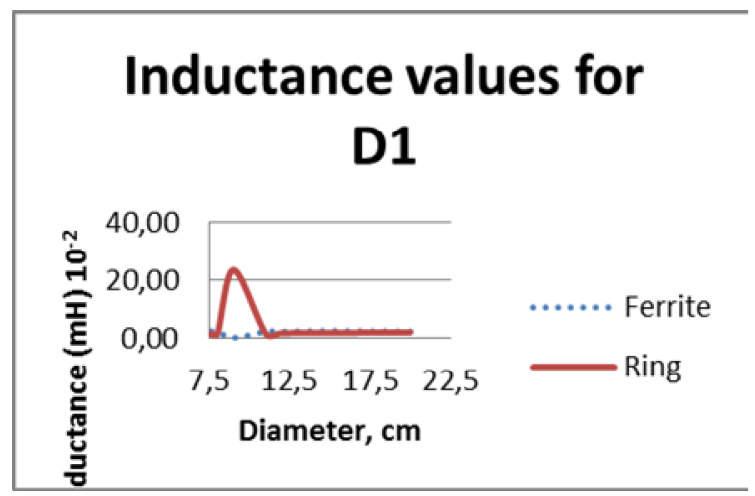

Fig. 9. Graph pattern for inductance values for ferrite bar and copper ring from D1

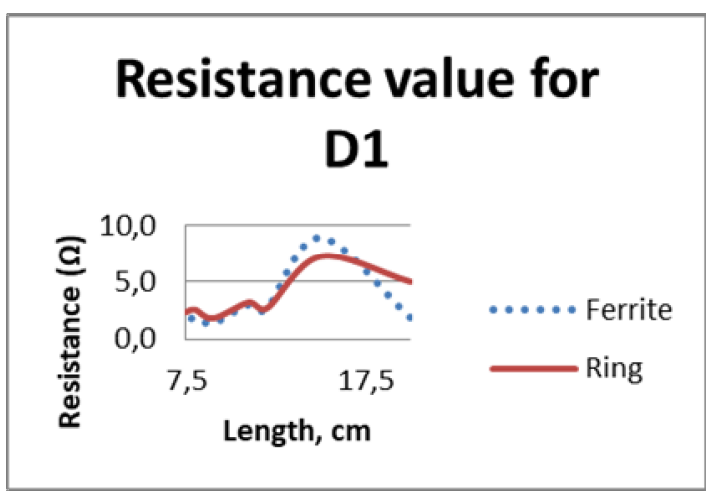

Fig. 10. Graph pattern for resistance values for ferrite bar and copper ring from $\mathrm{D}_{1}$

Table 7. Inductance, capacitance, resistance and voltage ${ }_{\mathrm{p}-\mathrm{p}}\left(\mathrm{V}_{\mathrm{p}-\mathrm{p}}\right)$ values of detector 2 (D2).

\begin{tabular}{cccc}
\hline Length of wire $(\mathbf{c m})$ & Inductance $(\boldsymbol{\mu H})$ & Resistance $(\mathbf{R})$ & Voltage $(\mathbf{m V})$ \\
\hline 0 & 2.53 & 0.00 & 0.0 \\
\hline 10 & 3.95 & 0.00 & 4.0 \\
\hline 20 & 4.00 & 1.10 & 17.2 \\
\hline 30 & 4.11 & 1.05 & 13.2 \\
\hline 40 & 4.50 & 0.88 & 18.3 \\
\hline 50 & 4.48 & 0.78 & 22.4 \\
\hline 60 & 4.80 & 0.58 & 23.8 \\
\hline 70 & 4.97 & 0.67 & 24.6 \\
\hline 80 & 5.15 & 0.70 & 25.7 \\
\hline 90 & 5.18 & 0.78 & 27.7 \\
\hline 100 & 5.26 & 0.90 & \\
\hline
\end{tabular}




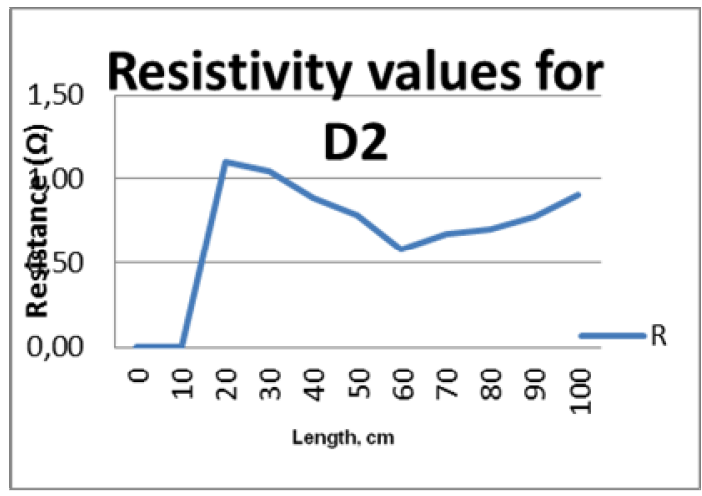

Fig. 11. Graph pattern resistivity values from D2

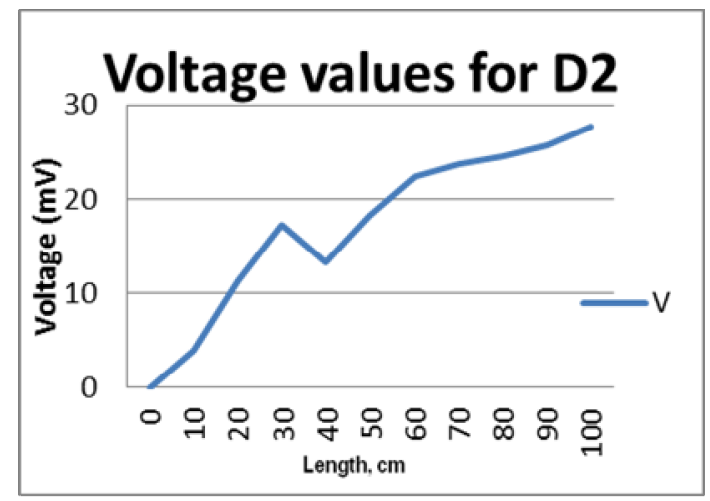

Fig. 12. Graph pattern Vp-p Values from D2

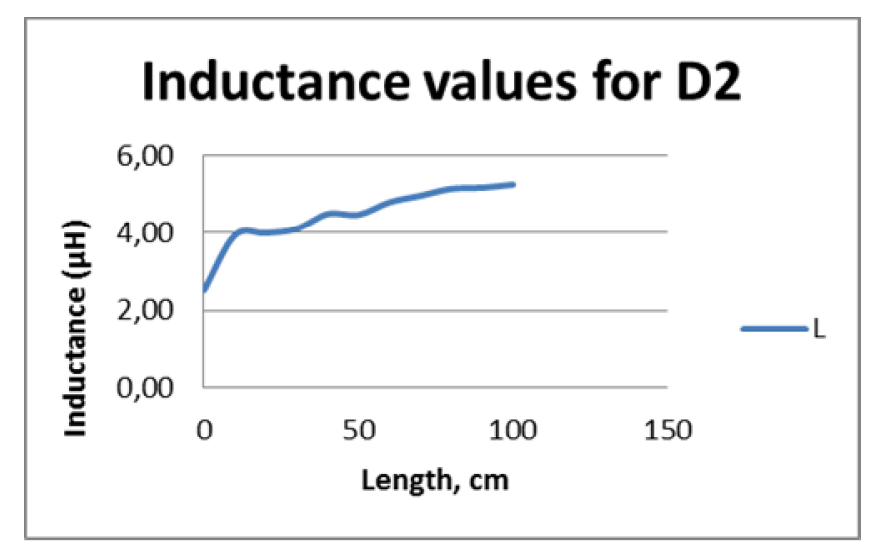

Fig. 13. Graph pattern for inductance values from D2

The results of magnetic after-effect lapsed time measurements conducted in this work shows that D1 had a higher lapsed time than D2. It took more than 15 minutes to have Vp-p decreased to zero value when the EM wave was switched off. It significantly affected the performance of the D1 even though it had a high sensitivity. D2 took 2 seconds to return to a zero voltage. One important mechanism to result in a short lapsed time for detector is to avoid incoming continuous electromagnetic wave to pile up.

\section{Conclusions}

We have constructed a novel ferrite-based EM wave detector for SBL application. From the two experiments conducted, we found that the sensitivity of D1 was much higher than D2 by $61 \%$. On the other hand, D2 had a lower lapsed time than D1, making it an important aspect for SBL application.

\section{Acknowledgement}

The author wishes to thank the SBL group of Universiti Teknologi PETRONAS for providing the facilities and all colleagues who have previously provided technical support. We appreciate ACME Ferrite Products Sdn. Bhd who has given support in provideing Ferrite products used in this experiment.

\section{References}

[1] T. Eidesmo, S.Ellingsrud, L.M.Macgregor, S.Constable, M.C Sinha, S.Johansen, H.Westerdahl, and F.N Kong. Remote detection of hydrocarbon filled layers using marine controlled source electromagnetic sounding. EAGE 64th Conference \& Exhibition, Florence, Italy (2002).

[2] GEO ExPro (June 2004) The same PRINCIPLE as in borehole logging (pp. 28-30).

[3] X C.Halfdan, GEO ExPro Proving the concept(June 2004). 
[4] F. Keisuke. High-Responsive Inductive Electromagnetic Sensor (Sept 2003), IEEE Transactions on Magnetic, Vol. 39, No. 5.

[5] A.T Daniel \& A.T James. Electromagnetic detection of a 410-km-deep melt layer in the Southwestern United States (pp. 991-994), Nature (21 June 2007)).

[6] H. Huang and I.J. Won Electromagnetic detection of buried metallic objects using quad-quad conductivity. GEOPHYSICS VOL. 69. NO 6.

[7] M.N.O Sadiku. Elements of electromagnetic. (pp. 336) Oxford University Press, New York. (2001).

[8] L.L Adams, T.P Cox, A.F James, A.M Richard, L.W Thompson. Electromagnetic wave logging system for determining resistivity and dielectric constant of earth formations. U.S Patent Documents: 2900595, 3900879, 3944910, 3993944 and 4012689

[9] Fawwaz T. Ulaby Electromagnetic for engineers. (pp.16). Pearson International Edition, New Jersey. (2005) .

[10] T. Eidesmo, S.Ellingsrud, L.M.Macgregor, S.Constable, M.C Sinha, S.Johansen, H.Westerdahl, and F.N Kong. Sea Bed Logging (SBL), a new method for remote and direct identification of hydrocarbon filled layers in deepwater areas. (pp.144-152) First Break Vol.20.3 (2002).

[11] Noorhana Y, A. Salwani., M.Z Hasnah, D. Hanita, A. A. Azizuddin and H. Hasnidar _ (2008) . Fabrication and phase, morphology and magnetic characterisation of Nickel Zink ferrite as an electromagnetic detector. Unpublished (2008). 